\title{
Use of the Sport Concussion Assessment Tool 5 (SCAT5) in professional hockey, part 2: which components differentiate concussed and non- concussed players?
}

\author{
Jared M Bruce, ${ }^{1}$ Joanie Thelen, ${ }^{2,3}$ Willem Meeuwisse, ${ }^{4}$ Michael G Hutchison, ${ }^{5}$ \\ John Rizos, ${ }^{6}$ Paul Comper, ${ }^{7}$ Ruben J Echemendia (1) ${ }^{8,9}$
}

- Additional material is published online only. To view please visit the journal online (http://dx.doi.org/10.1136/ bjsports-2020-102072).

For numbered affiliations see end of article.

Correspondence to Dr Ruben J Echemendia, UOC Concussion Care Clinic, State College, Pennsylvania, USA; rechemendia@comcast.net

Accepted 21 July 2020

- http://dx.doi.org/10.1136/ bjsports-2020-10207

Check for updates

(c) Author(s) (or their employer(s)) 2020. No commercial re-use. See rights and permissions. Published by BMJ.

To cite: Bruce JM, Thelen J, Meeuwisse W, et al.

Br J Sports Med Epub ahead of print: [please include Day Month Year]. doi:10.1136/ bisports-2020-102072

\begin{abstract}
Objectives To examine the utility of Sport Concussion Assessment Tool (SCAT5) subcomponents in

differentiating physician diagnosed concussed players from controls.

Methods We evaluated 1924 professional hockey players at training camp using the National Hockey League (NHL) Modified SCAT5 prior to the 2018-2019 season. Over the course of the season, 314 Englishspeaking players received SCAT5 evaluations within 1 day of a suspected concussive event. Of these players, $140(45 \%)$ were subsequently diagnosed with concussion by their team physicians.

Results Concussed players reported more symptoms (Concussed M=8.52, SD=4.78; Control M=3.32, $S D=3.97)$, and recalled fewer words than Controls on both the Immediate Memory (Concussed $M=19.34$, $S D=4.06$; Control $M=21.53, S D=2.94$ ) and Delayed Recall (Concussed $z=-0.91$; Control $z=-0.09$ ) tasks during the acute evaluation. Concussed players also made more errors than Controls on the mBESS and were more likely to report double vision and exhibit clinicianobserved balance problems than controls. There were no between-group differences on the Concentration component of the SCAT5. Stepwise regression revealed that symptom report and list learning tasks both accounted for independent variance in identifying players diagnosed with concussion.
\end{abstract}

Conclusions These findings provide support for use of the SCAT5 to assist in identifying concussed professional hockey players. When examining SCAT5 subtests, both symptom report and the 10-item word list accounted for independent variance in identifying concussion status in this sample of professional hockey players. The mBESS also differentiated Concussed players and Controls. The Concentration component of the SCAT5 did not significantly differentiate Concussed players and Controls.

\section{INTRODUCTION}

The process for diagnosing sports-related concussion (SRC) and subsequent management of the injury has been described as one of the most challenging tasks in sports medicine. ${ }^{1}$ The diagnostic challenge is related to the pathophysiology of concussion, ${ }^{2}$ which is complicated by factors such as non-specific concussion symptoms, variable timeframe for symptom onset, imprecise thresholds for symptom detection and symptom duration, dynamic and changing symptoms over time, lack of definitive biomarkers and lack of clarity in the definition of concussion. ${ }^{3-6}$ The complexity and lack of definitive diagnostic markers results in an imperfect 'gold standard' that is based on clinical diagnosis made by a qualified healthcare professional. ${ }^{56}$

First published in 2004 by the Concussion In Sport Group (CISG), the Sport Concussion Assessment Tool $\left(\mathrm{SCAT}^{7}\right.$ was designed as an educational and clinical tool to help standardise the evaluation of SRC. Subsequent revisions were published

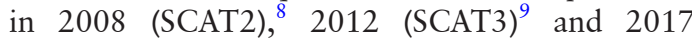
(SCAT5). ${ }^{10}$ Note that there is no SCAT4. The most recent iteration was named the SCAT5 to align all of the SCAT tools (SCAT5, child SCAT5 and Concussion Recognition Tool5) with the fifth meeting of the CISG. The SCAT tools were not intended to 'diagnose' concussion but rather to provide a framework for multidimensional assessment of SRC, the results of which may be used as part of a diagnostic formulation.

To improve the psychometric properties of the SCAT5 clinicians have the option of choosing between 5 -word and 10-word lists in the Immediate Memory and Delayed Recall tasks. Previous versions only employed a five-word list that exhibited substantial ceiling effects. ${ }^{11}$ Ceiling effects reduce the sensitivity to subtle changes in test performance.

The SCAT5 also included changes to the administration instructions for the symptom checklist. Rather than asking athletes how they currently feel at both baseline and acute injury evaluations as done in the SCAT3, in the SCAT5 athletes are asked to report their typical symptoms during the baseline assessment and their current symptoms at the acute postinjury assessment, thus differentiating trait (long-term) and state (short-term) symptom expression, ${ }^{12}{ }^{13}$ respectively. Additionally, to assess deficits in rudimentary reading ability, athletes are asked to read the instruction paragraph aloud before rating their symptoms. This information is incorporated into the Neurological screening to help identify dyslexia and other receptive/expressive language disturbances.

The clinical components of the SCAT5 examined in this study were: symptom evaluation, Immediate Memory, Concentration (Digits Backward; Months in Reverse), Neurological screen, balance 
examination (modified Balance Error Scoring System; mBESS) and Delayed Recall. Our aim was to examine how the various SCAT5 subcomponents fared in distinguishing physician diagnosed concussed and non-concussed professional hockey players.

\section{METHODS}

Study design

As part of the National Hockey League (NHL)/ National Hockey League Players' Association (NHLPA) concussion evaluation and management programme, all players attending preseason training camps prior to the 2018-2019 season ('baseline testing') completed the NHL Modified SCAT5 electronic application (briefly, the App). Our study uses data collected from that programme. Once the season got under way, players in an NHL game or practice who were suspected of having a concussion and/or exhibited visible signs of possible concussion (eg, slow to get up, clutching of head, balance problems, blank stare, motionlessness) ${ }^{14}$ following a direct or indirect blow (indirect transmission of forces from the torso) to the head underwent a repeat evaluation for possible concussion. Evaluation included the SCAT5 App and clinical examination administered by the team physician and/or athletic trainer. As noted above, in the absence of an objective biomarker or empirically validated tool for diagnosis of SRC, the 'gold standard' is a clinical determination made by a clinician using established standards. ${ }^{1}$ The CISG definition of $\mathrm{SRC}^{4}$ was used by NHL team physicians to arrive at a concussion diagnosis.

Players who were evaluated and not diagnosed with concussioncomprised the Control group. Like players diagnosed with a concussion, these Controls were typically evaluated during or immediately after a game-thus accounting for the possible influence of physical activity, secondary injury (eg, ankle sprain) and test-taking motivation.

\section{Inclusion criteria}

Researchers extracted player baseline and initial prospective postinjury data from an NHL SCAT5 database. We only extracted data for initial (first time) SCAT5 suspected injury evaluations. Players who had multiple evaluations for suspected concussion or multiple prospective diagnosed concussions were not included, which increased the likelihood that all players in the analyses had similar exposure to the SCAT5 at the acute evaluation and ensured that each case in the database was unique. Note that even though only first time SCAT5 suspected injury evaluations were included, many of these players had a prior history of concussion. Data were extracted if the player was English-speaking and had valid baseline and acute SCAT5 evaluations (within 1 day of injury). Lastly, mBESS analyses were restricted to players tested without skates due to our work ${ }^{15}$ showing marked ceiling effects on mBESS with players wearing skates. Figure 1 shows an inclusion flowchart for the study.

\section{Measures}

The NHL modified SCAT5 was adapted for use in the NHL and was administered via a custom App, which reproduces all components of the original SCAT5 with modifications to enhance the standardised administration of the instrument by audibly presenting the Immediate Memory trials and Digits Backward at the rate of one item per second. Players chose from eight different languages (English, Swedish, French, Russian, Czech, Finnish, Slovak or German) to endorse symptoms on the PostConcussion Symptom Scale (PCSS). ${ }^{15}$ The rest of the examination was administered in English. Lastly, countdown timers were added to all subtests requiring a timed component (eg, Delayed Recall, balance measures) and progression from one subtest to the next was dependent on completion of prior subtests. No significant performance differences were found between paper administration and App administration as part of a large normative sample at baseline. ${ }^{15}$

\section{Data analytical strategy}

Between-group differences in age and time between baseline/ acute evaluations were examined with t-tests. Due to the presence of non-normal distributions and/or outliers common in clinical populations, non-parametric analyses were used for hypothesis testing. Between-group differences on the SCAT5 at baseline were examined using Mann-Whitney U tests. Betweengroup differences at the acute evaluation were examined using Quade's nonparametric Analysis of Covariance controlling for baseline performance. Between group SCAT5 differences in change from baseline to acute evaluation were examined with Mann-Whitney U tests. Follow-up Wilcoxon tests were used to examine within group changes from baseline to acute evaluation. Non-parametric effect sizes were examined using the probability of concordance (Area Under the Curve, AUC), bootstrapped 95\% CIs (1000 samples) of median values, ORs and $r$ values. Sensitivity, specificity, positive predictive value (PPV) and negative predictive value (NPV) were calculated for a series of cut points that were determined using receiver operating characteristic (ROC) curves to calculate Youden's statistic and common normative classification thresholds (15th and 5 th percentile). Forward stepwise regression examined which components of the SCAT5 accounted for independent variance in concussion diagnosis. Immediate Memory scores and Delayed Recall scores were corrected for form differences as described previously. ${ }^{14}$ IBM SPSS V.22, Microsoft Excel and Medcalc.org were used for analyses. Significance levels were set at $\mathrm{p}<0.05$.

\section{RESULTS}

A total of 1924 players received baseline SCAT5 testing with 314 players meeting criteria to have their data extracted from the NHL database. Of these, 140 players were diagnosed with concussion (Concussion group), and 174 were evaluated for possible concussion but not diagnosed with concussion (Control group). Mean time from suspected injury to SCAT5 administration (acute SCAT5) was $0.12( \pm 0.32)$ days. Of the extracted data files, we know that 167 Controls and 111 Concussed players were tested the same day as the suspected injury and the remaining players were tested the day following the suspected injury. $\mathrm{X}^{2}$ analyses revealed Concussed players were significantly more likely to be tested on the day following the event $\left(\chi^{2}=21.29, \mathrm{df}=1, \mathrm{p}<0.001\right.$, OR $6.23,95 \%$ CI 2.64 to 14.72 ). Mean time between baseline SCAT5 and acute SCAT5 was $95.23( \pm 53.51)$ days. An independent samples t-test revealed no significant group differences between Controls and Concussed players in time between baseline and acute testing, $\mathrm{t}(312)=-0.44, \mathrm{p}=0.662$, mean difference $=-2.66$, $95 \% \mathrm{CI}-14.63$ to 9.307 . Mean age at baseline evaluation was $25.49 \pm 4.04$ years, with no significant between-group age differences, $\mathrm{t}(319)=.28, \mathrm{p}=0.78$, mean difference $=0.13,95 \% \mathrm{CI}-0.77$ to 1.04 . No significant differences were found between Concussed (69\% one or more concussions) and Control (59\% one or more concussions) groups' history of previous concussion, $\chi 2=3.42$, $\mathrm{p}=0.065$, OR $1.55,95 \%$ CI 0.97 to 2.46 ).

\section{Baseline results}

Table 1 presents baseline descriptive, significance testing and effect size statistics for Concussed and Control players. 


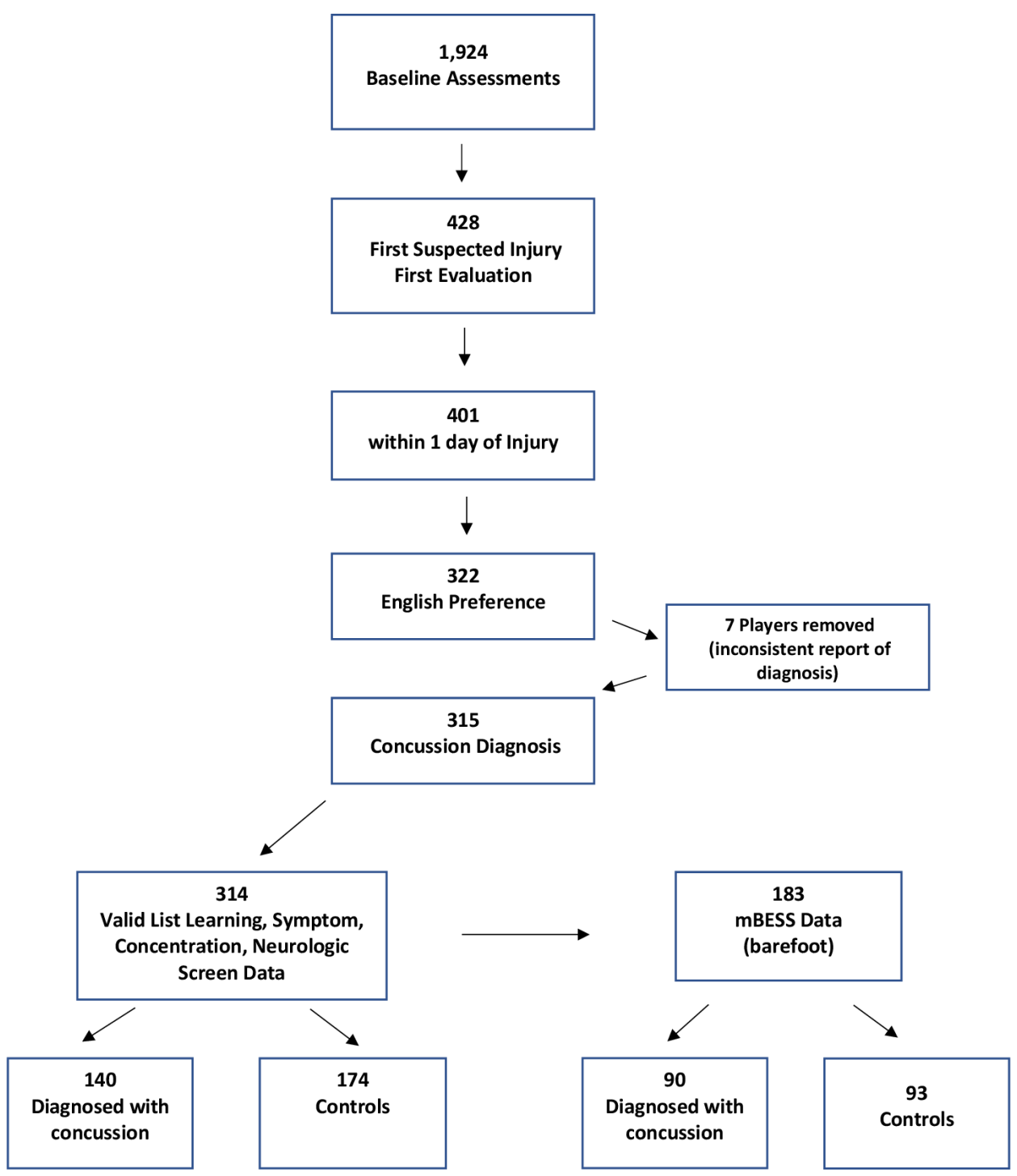

Figure 1 Participant inclusion flow chart. mBESS, modified Balance Error Scoring System.

Mann-Whitney U tests revealed that Concussed players reported more symptoms and performed more poorly on baseline SCAT5 Delayed Recall when compared with Controls. Significant between group differences at baseline revealed AUC 95\% CIs between 0.53 and 0.67 . A non-significant trend also suggested Concussed players made more mBESS balance errors than Controls at baseline, with AUC 95\% Cis between 0.50 and 0.67. No significant differences were found between groups on corrected Immediate Memory and Concentration (see table 1).

\section{Baseline/suspected injury evaluations}

Table 2 presents acute injury descriptive, significance testing and effect size statistics. Non-parametric Quade's Analysis of Covariance Controlling for baseline performance revealed that Concussed players endorsed more symptoms, made more balance errors on the mBESS and performed worse on Immediate Memory and Delayed Recall when compared with Controls. Probability of concordance 95\% CIs were between 0.77 and 0.88 for symptom report measures and between 0.56 and 0.72 for statistically significant behavioural measures. No significant difference was found between groups on the Concentration composite (Digit Span and Months in Reverse) although a significant difference emerged when Digit Span was assessed in isolation (AUC 95\% CI 0.50 to 0.63 ).

Table 3 shows difference scores for each of the SCAT5 indices for Controls and Concussed players. Mann-Whitney U tests revealed increased symptoms and worse memory performance from baseline to acute evaluation among Concussed players when compared with Controls. Despite a larger change in performance among Concussed players, follow-up Wilcoxonsigned rank tests revealed that both Controls and Concussed players endorsed more total symptoms (Concussed $r=0.84$; Controls $r=0.60$ ) and greater symptom severity (Concussed $r=0.86$; Controls $r=0.60$ ) at the acute evaluation than the baseline evaluation (all p <0.001). Similarly, both Concussed players $(p<0.001, r=0.42)$ and Controls' $(p=0.019, r=0.18)$ exhibited a decline in Delayed Recall performance from baseline to acute evaluation. In contrast, Concussed players' performance on Immediate Memory declined from baseline to acute evaluation $(p<0.001, r=0.45)$ while no significant change was found among Controls $(p=0.350, r=0.07)$, althoughConcussed players showed significantly greater decline on Immediate Memory from baseline to acute evaluation. No significant between group change score differences were noted on the 


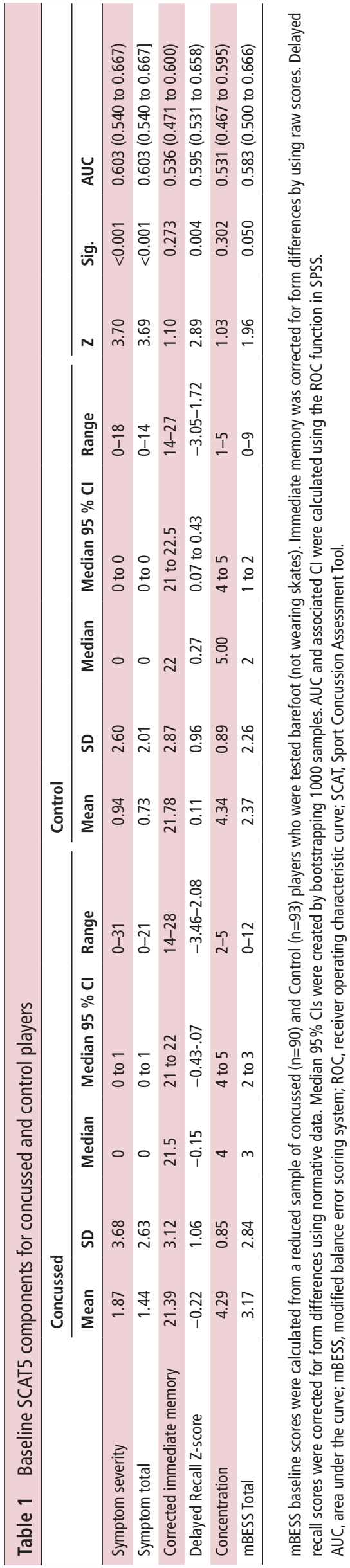

mBESS or Concentration composite. Exploratory follow-up analyses revealed that neither concussed players nor Controls had significant declines from baseline to acute evaluations on the mBESS (Concussed, $\mathrm{p}=0.105, \mathrm{r}=0.17$; Controls, $\mathrm{p}=0.760$, $\mathrm{r}=0.03$ ) or Concentration composite (Concussed, $\mathrm{p}=0.138$, $r=0.13$; Controls, $p=0.636, r=0.04)$.

$\mathrm{X}^{2}$ analyses examined the individual components of the Neurologic screen. Concussed players were more likely to report double vision $(10 / 140)$ than Controls $\left(3 / 174, \chi^{2}=5.74\right.$, $\mathrm{p}=0.017, \quad \mathrm{OR}=4.39,95 \%$ CI 1.18 to 16.25$)$. Similarly, Concussed players (17/140) were more likely to have clinicianobserved balance problems than Controls on the Neurologic screen $\left(8 / 174, \chi^{2}=6.03, p=0.014, \mathrm{OR}=2.87,95 \%\right.$ CI 1.20 to 6.86). No significant group differences were observed for other Neurologic screen components, including Finger to Nose (Concussed $=3 / 140$, Control $=0 / 174, \chi^{2}=3.77, p=0.052$ ), or Cervical examination (Concussed $=20 / 140$, Control $=17 / 174$, $\chi^{2}=1.52, p=0.217$, OR $1.54,95 \%$ CI 0.77 to 3.07$)$. One concussed player had difficulty reading the symptom instruction paragraph (Concussed $=1 / 140$, Control $=0 / 174)$.

\section{Exploratory cut-point/follow-up analyses}

Stepwise logistic regression was conducted with baseline symptoms and memory performance entered in the first block (due to group baseline differences on these measures) and acute symptoms, significant neurological examination variables, and acute memory performance entered stepwise in the second block. Both acute symptom severity (OR 1.12; 95\% CI 1.09 to 1.16 , $\mathrm{p}<0.001)$ and acute Immediate Memory (OR 0.87; 95\% CI 0.80 to $0.95, \mathrm{p}=0.002$ ) predicted independent variance in concussion status after accounting for baseline symptoms and baseline memory performance. Follow-up logistic regression with baseline behavioural measures entered in the first block and acute behavioural measures entered stepwise in the second block in the subset of patients with mBESS data revealed that both Immediate Memory (OR $0.87,95 \%$ CI 0.79 to $0.95, p=0.002)$ and mBESS (OR 1.14, 95\% CI 1.02 to $1.27, \mathrm{p}=0.013$ ) accounted for independent variance in concussion status.

Figure 2A shows ROC curves for acute symptom severity, total symptoms, Delayed Recall and Immediate Memory. Figure 2B shows a ROC curve for mBESS among the subset of patients with formal balance testing. Online supplementary figures 1-18 compare frequency distributions between concussed players and Controls for primary behavioural performance and self-report measures. Of note, a handful of Controls showed very poor Delayed Recall at the acute evaluation, as seen in online supplementary figure s14. Examination of the data showed that $81 \%$ of players who recalled three or fewer words on the Delayed Recall measure were diagnosed with concussion (25/31). Three players who were not diagnosed with concussion recalled one or zero words at the acute evaluation. Table 4 presents the sensitivity, specificity, PPV and NPV for various cut-points for performance on statistically significant behavioural and self-report portions of the SCAT5.

\section{DISCUSSION}

This study examined the ability of SCAT5 subcomponents to differentiate concussed from non-concussed athletes in a sample of professional hockey players. Results suggest that symptoms and 10-word Immediate Memory and Delayed Recall tasks differentiate concussed hockey players and Controls. Moreover, results show that Immediate Memory adds predictive value above symptoms alone. Of note, at least three control players 


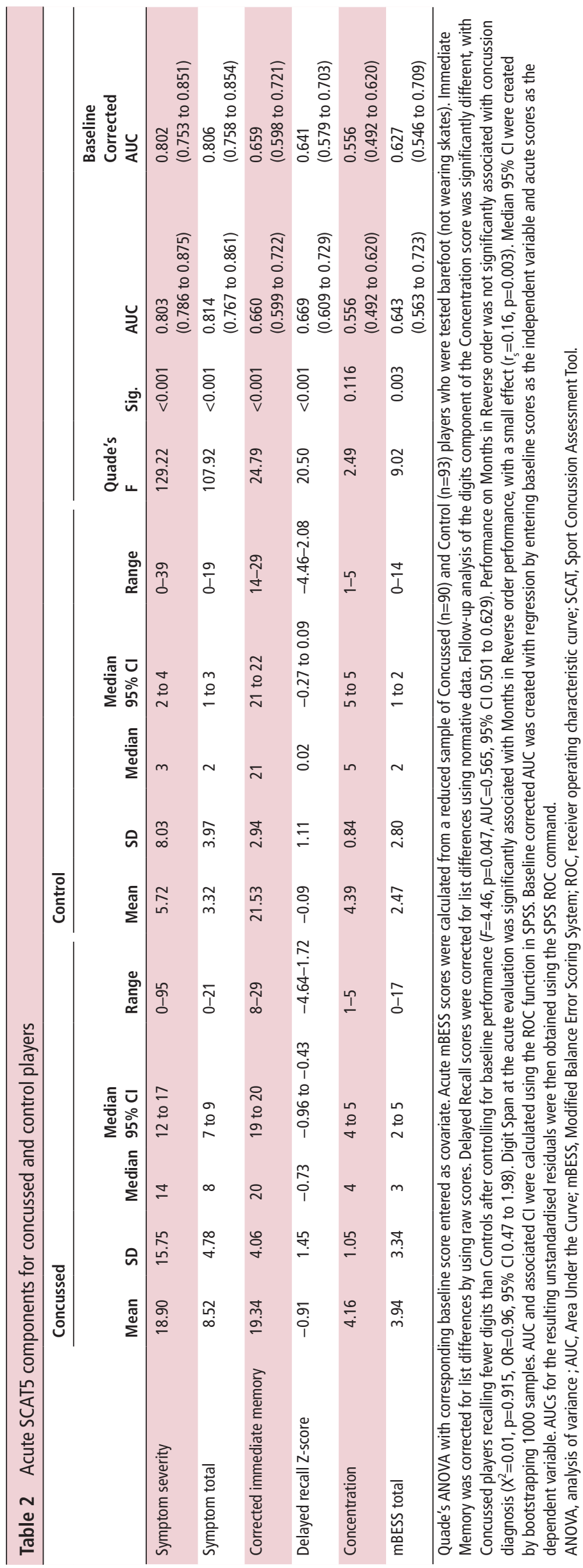



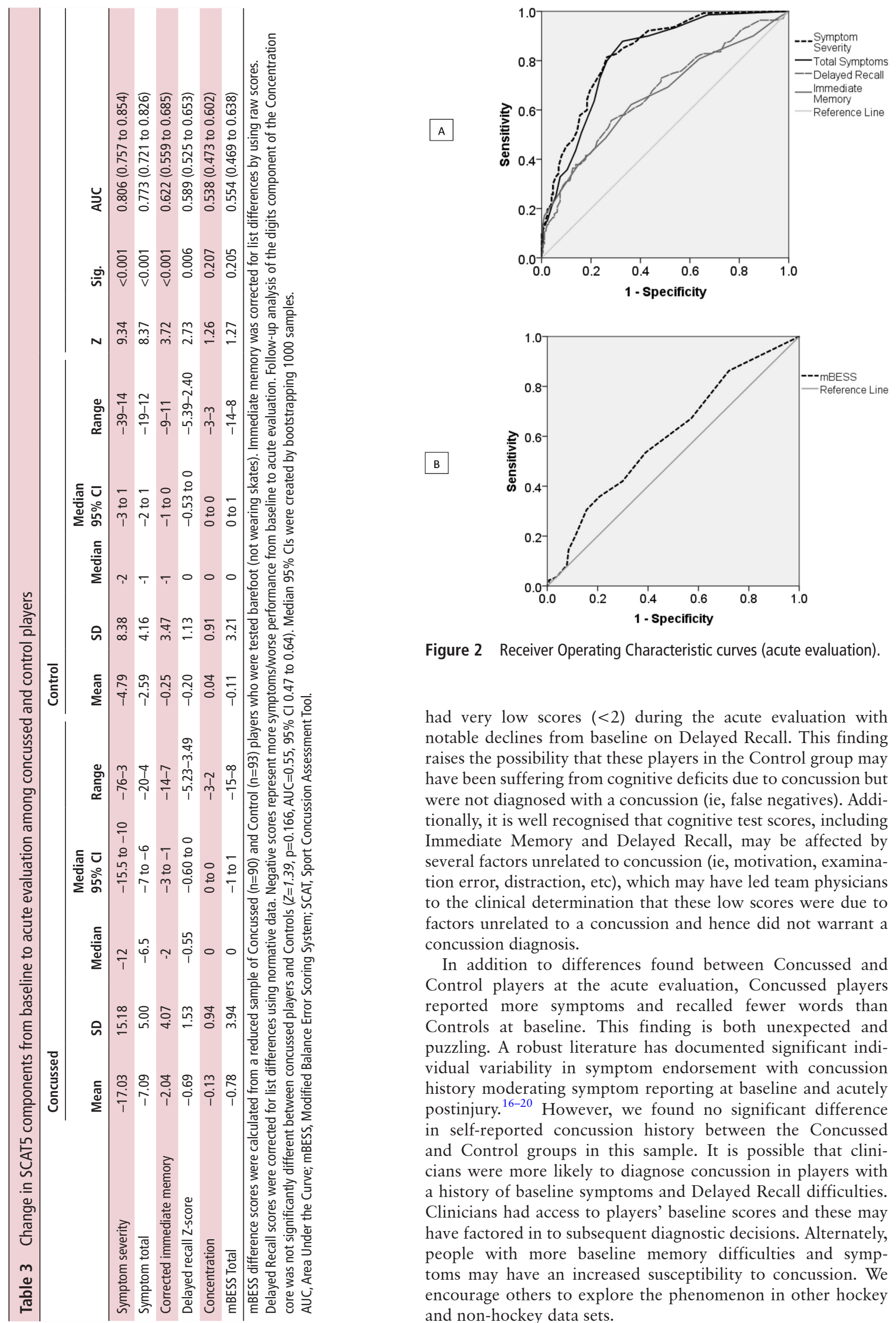

Figure 2 Receiver Operating Characteristic curves (acute evaluation).

had very low scores $(<2)$ during the acute evaluation with notable declines from baseline on Delayed Recall. This finding raises the possibility that these players in the Control group may have been suffering from cognitive deficits due to concussion but were not diagnosed with a concussion (ie, false negatives). Additionally, it is well recognised that cognitive test scores, including Immediate Memory and Delayed Recall, may be affected by several factors unrelated to concussion (ie, motivation, examination error, distraction, etc), which may have led team physicians to the clinical determination that these low scores were due to factors unrelated to a concussion and hence did not warrant a concussion diagnosis.

In addition to differences found between Concussed and Control players at the acute evaluation, Concussed players reported more symptoms and recalled fewer words than Controls at baseline. This finding is both unexpected and puzzling. A robust literature has documented significant individual variability in symptom endorsement with concussion history moderating symptom reporting at baseline and acutely postinjury ${ }^{16-20}$ However, we found no significant difference in self-reported concussion history between the Concussed and Control groups in this sample. It is possible that clinicians were more likely to diagnose concussion in players with a history of baseline symptoms and Delayed Recall difficulties. Clinicians had access to players' baseline scores and these may have factored in to subsequent diagnostic decisions. Alternately, people with more baseline memory difficulties and symptoms may have an increased susceptibility to concussion. We encourage others to explore the phenomenon in other hockey and non-hockey data sets. 
Table 4 Sensitivity, specificity, positive predictive value (PPV) and negative predictive value (NPV) for various normative cut points of acute Delayed Recall

\begin{tabular}{|c|c|c|c|c|}
\hline & $\begin{array}{l}\text { Probability of a positive test } \\
\text { in those with a concussion }\end{array}$ & $\begin{array}{l}\text { Probability of a negative test } \\
\text { in those without concussion }\end{array}$ & $\begin{array}{l}\text { Probability of having a } \\
\text { concussion if the test is } \\
\text { positive }\end{array}$ & $\begin{array}{l}\text { Probability of not having } \\
\text { a concussion if the test is } \\
\text { negative }\end{array}$ \\
\hline & Sensitivity & Specificity & PPV & NPV \\
\hline \multicolumn{5}{|l|}{ Immediate Memory } \\
\hline 20 (Youden) & $62.14 \%(53.56-70.20)$ & $63.79 \%(56.18-70.93)$ & $58.00 \%(52.17-63.61)$ & $67.68 \%(62.23-72.69)$ \\
\hline 17 (Borderline) & $29.57 \%(21.26-36.81)$ & $91 \%(86.18-95.09)$ & $73.73 \%(60.60-82.21)$ & $61.39 \%(58.65-64.06)$ \\
\hline 16 (Low) & $22.14 \%(15.57-29.92)$ & $95.40 \%(91.14-97.99)$ & $79.49 \%$ (64.79-89.08) & $60.36 \%(58.09-62.59)$ \\
\hline \multicolumn{5}{|l|}{ Delayed Recall } \\
\hline 32nd \%ile (Youden) & $55.71 \%(47.08-64.10)$ & $71.84 \%$ (64.53-78.38) & $61.42 \%(54.62-67.80)$ & $66.84 \%(62.09-71.28)$ \\
\hline 15th \%ile (Borderline) & $41.43 \%(41.43)$ & $82.18 \%(75.68-87.56)$ & $65.17 \%(56.25-73.13)$ & $63.56 \%(59.88-67.08)$ \\
\hline 5th \%ile (Low) & $27.86 \%(20.62-36.06)$ & $91.95 \%(86.87-95.53)$ & $73.58 \%(61.20-83.11)$ & $61.30 \%(58.62-63.92)$ \\
\hline \multicolumn{5}{|l|}{ mBESS } \\
\hline 5 (Youden) & $40.00 \%(29.81-50.87)$ & $86.02 \%(77.28-92.34)$ & $73.47 \%$ (61.17-82.96) & $59.70 \%(55.12-64.12)$ \\
\hline 6 (Borderline) & $34.44 \%(24.74-45.20)$ & $91.40 \%(83.75-96.21)$ & $79.49 \%(65.32-88.85)$ & $59.03 \%(55.06-62.89)$ \\
\hline 9 (Low) & $6.67 \%(2.49 \%-13.95 \%)$ & $95.70 \%(89.35-98.82)$ & $60.00 \%(30.45-83.71 \%)$ & $51.45 \%(49.69-53.19)$ \\
\hline \multicolumn{5}{|l|}{ Total symptoms } \\
\hline 4 (Youden) & $87.86 \%$ (81.27-92.76) & $67.24 \%(59.73-74.15)$ & $68.33 \%(63.36-72.92)$ & $87.31 \%$ (81.33-91.58) \\
\hline 3 (Borderline) & $90.00 \%(83.79-94.42)$ & $56.90 \%(49.19-64.37)$ & $62.69 \%(58.40-66.78)$ & $87.61 \%(80.89-92.20)$ \\
\hline 6 (Low) & $72.86 \%(64.70-80.02)$ & $76.44 \%(69.42-82.53)$ & $71.33 \%(65.14-76.81)$ & $77.78 \%$ (72.49-82.29) \\
\hline \multicolumn{5}{|l|}{ Symptom severity } \\
\hline 8 (Youden) & $81.43 \%(73.98-87.50)$ & $73.56 \%$ (66.36-79.95) & $71.25 \%(65.64-76.27)$ & $83.12 \%(77.48-87.57)$ \\
\hline 4 (Borderline) & $92.14 \%(86.38-96.01)$ & $56.90 \%(49.19-64.37)$ & $63.25 \%(59.02-67.26)$ & $90.00 \%(83.42-94.15)$ \\
\hline 7 (Low) & $82.86 \%(75.58-88.70)$ & $69.54 \%(62.12-76.28)$ & $68.64 \%(63.33-73.50)$ & $83.45 \%(77.57-88.03)$ \\
\hline
\end{tabular}

Younden cutpoints derived using Youden's Index. Borderline and low performance cut-points based on NHL normative data for the 15th and 5th percentile, respectively.

NPV, Negative Predictive Value; PPV, Positive Predictive Value.

\section{The role of memory}

Memory performance differentiated Concussed and Control athletes postinjury even after controlling for group differences at baseline. Taken together, these data point to the added value of memory tasks when identifying physician diagnosed concussed players and thereby underscores the role of these cognitive tasks in making diagnostic decisions. The robust nature of these findings suggest that medical staffs should be keenly aware of the significance of an unusually low score on memory measures both at baseline and acute evaluation. Although low Immediate Memory and Delayed Recall scores are not pathognomonic for concussion, these results suggest that players with Delayed Recall scores below the 16th percentile and Immediate Memory scores below 18 at the acute evaluation should be very carefully examined with clinicians encouraged to err on the side of player safety in making (or excluding) a diagnosis of concussion.

These data also suggest that use of the 10-item word list is preferred over the 5 -item word list among players in this sample. Our data reveal that professional hockey players with an English language preference $(n=1123)$ generated significant ceiling effects on the five word lists with $72 \%$ of players achieving a perfect score (see online supplementary tables S7-S10). In contrast, the 10 -word list was largely normally distributed (S9) with only one player $(0.1 \%)$ achieving a perfect score. A similar pattern was observed on Delayed Recall (online supplementary tables S8 and $\mathrm{S} 10$ ). The increased challenge posed by the 10 -word lists appear better suited to capture more subtle learning and recall deficits. Though some work shows the 5-item list has value in differentiating Concussed and Control athletes, ${ }^{21}$ other work indicates the 5 -item list has limited discriminative ability ${ }^{22}$ - especially when given more than 2 days postinjury. The current study found that the 10 -item list adds independent value as part of the SCAT5 when given within 1 day of injury. Additional work is needed to determine whether the 10 -item word list can discriminate concussed athletes from controls two or more days following concussive events and across different language groups.

In isolation from baseline performance, Delayed Recall scores differentiated Concussed players and Controls. Close inspection of individual level data revealed some players who showed notable improvement at their acute evaluation, including one player who recalled three words at baseline and nine at acute evaluation. Since it is highly unlikely that concussion improves memory performance, this level of performance raises the possibility of incomplete effort or performance invalidity ${ }^{23} 24$ at baseline thereby reducing the power of change score analyses. At the group level, the present study found no significant benefit of using change scores over acute scores-with effect sizes for acute scores typically being somewhat larger. This finding has implications for the value of baseline testing and the potential need to develop embedded markers of performance validity ${ }^{24}$ when giving the SCAT5 as part of a baseline/postinjury protocol. Additional research is needed to determine the value of baseline SCAT5 testing across diverse populations. Although decline on the SCAT5 does not appear to add unique predictive ability to concussion diagnosis in logistic regression analyses, various cut points for the acute evaluation were produced that showed very good specificity and acceptable PPV (see table 4). Of note, the obtained PPVs and other metrics would be improved if the three players who had scores suggestive of severe memory impairment at the acute evaluation had also been diagnosed with concussion.

\section{The role of the mBESS}

In addition to group memory differences at the acute evaluation, we also found significant between group difference on the 
mBESS at the acute evaluation. In contrast, change in mBESS performance was not significantly different between groups. This mixed finding suggests the possibility of additional testing error being introduced on the mBESS when making use of both baseline and acute results. Similar to our memory findings, examination of supplemental tables shows several players who demonstrated notable balance difficulties at the acute evaluation who were not diagnosed with concussion. Again, there are many reasons a player could have balance problems independent of concussion, foremost of which may be orthopaedic injury. Nonetheless, our results suggest that caution is warranted when players have more than six mBESS errors. It is also important to reiterate that this analysis was conducted on a smaller sample of players, as many players were assessed acutely while wearing skates and could not be tested with the mBESS. ${ }^{15}$ Future research should examine the independent value of the mBESS in sports that are more amenable to acute balance assessment in bare feet or conventional footwear.

\section{The role of concentration}

Beyond recognising the value of the symptom report, balance and memory scales from the NHL modified SCAT5, it is also worth mentioning that one subsection did not add independent value in differentiating Concussed and Control groups . Specifically, the Concentration composite of the SCAT5 did not significantly differentiate Concussed athletes from Controls and had smaller effects than those obtained by the other behavioural performance measures. As noted in our prior work, ${ }^{15}$ this may be due in part to ceiling effects (see online supplementary tables S11,S12), particularly with regard to Months in Reverse. As such, eliminating or replacing Months in Reverse with another working memory task and increasing the number of digit strings in the Digit Backward subtest would make the tests more difficult, possibly increasing the instrument's sensitivity to concussion. Follow-up analyses revealed mixed findings for Digit Backward used in isolation-with Concussed players performing worse acutely (see table 2 note), but no significant differences in decline from baseline. Months in Reverse was not significantly associated with concussion diagnosis and only minimally associated with Digit Backward performance at the acute evaluation $\left(r_{s}=0.16, p<0.01\right)$. These findings bring into question the use of Months in Reverse as part of the SCAT5 as well as the combined use of Digits Backwards and Months in Reverse as a composite index.

\section{Role of neurological examination}

The Neurological screening component of the SCAT5 draws from information gathered as part of other subcomponents of the instrument. Concussed players were more likely than Controls to have diplopia (double vision) diagnosed during the Neurologic screen. Diplopia had low sensitivity but an acceptable PPV of $77 \%$. This result highlights the role of oculomotor measures on the assessment of SRC and the potential of emerging products that allow for more sensitive and objective measures of vision abnormalities following concussion. ${ }^{2526}$ The present findings also support the value of clinician reported/observed balance problems as part of the Neurological screen. Consistent with protocols in other professional sports leagues, ${ }^{27}$ NHL team physicians are instructed to review video of suspected concussive events as part of their evaluations. It is possible that clinician-rated balance problems are, in part, based on observed motor incoordination that occurred immediately following the injury as well as motor difficulties observed on the formal balance measures.
Limitations and summary

This study has several limitations. As already noted, Controls were defined as players suspected of concussion, but not diagnosed with a concussion. Although this approach has several advantages, some players designated as Controls may have been actually concussed yet not diagnosed as such (false negatives). However, as noted above, the lack of pathognomonic biomarkers in the diagnosis of concussion makes this a limitation of the field rather than this study per se. Similarly, although team physicians were instructed not to consider SCAT5 subcomponent performance when making the determination of a concussion diagnosis, they were nonetheless aware of the players' results, which could have influenced their decisionmaking, falsely increasing the metrics of the SCAT5 examined in this project. Additional studies with physicians blinded to the SCAT5 data are needed, as are studies using controls without suspected concussion. Further, this study only examined English-speaking players; evaluation of this instrument among players with other primary languages is needed. Finally, although this study used a robust sample of professional hockey players, the results may not fully generalise to other populations, including females, collegiate and high school athletes, and players from different ethnic backgrounds. More research in these and other diverse populations is needed.

In summary, these results support the use of SCAT5 components in differentiating Concussed players and Controls in professional ice hockey during the acute phase of the injury (within 1 day of a suspected event). Results extend previous findings highlighting symptom report as an important component of SCAT5 evaluation and concussion diagnosis. Both symptom report and memory performance account for independent variance in concussion diagnosis. Future iterations of the SCAT should consider increasing the difficulty of the Concentration subtests to avoid ceiling effects and improve test sensitivity, and to include embedded measures of performance validity. We contend that studies that examine the utility of the SCAT5 at different points post-injury are critically important.

\section{What are the findings?}

- First study to examine whether components of the Sport Concussion Assessment Tool 5 (SCAT5) could differentiate Concussed professional hockey players from Controls.

- List-learning/memory and symptom report show independent contributions to the identification of players diagnosed with concussion.

- Concussed players were more likely to report double vision and exhibit clinician-observed balance problems than ontrols on the SCAT5 Neurological screen.

How might it impact on clinical practice in the future?

- These data underscore the independent value of symptom report and cognitive assessment measures in concussion evaluation.

- The results provide evidence for use of the 10-item word list as part of SCAT5 administration.

- The data raise questions about the need to assess the validity/accuracy of player performance during baseline testing. 


\section{Author affiliations}

'Department of Biomedical and Health Informatics, University of Missouri Kansas City School of Medicine, Kansas City, Missouri, USA

${ }^{2}$ National Hockey League, New York City, New York, USA

${ }^{3}$ University of Missouri Kansas City, Kansas City, Missouri, USA

${ }^{4}$ National Hockey League, Calgary, Alberta, Canada

${ }^{5}$ Faculty of Kinesiology and Physical Education, University of Toronto, Toronto, Ontario, Canada

${ }^{6}$ Department of Family and Community Medicine, University of Toronto, Toronto, Ontario, Canada

${ }^{7}$ Department of Family and Community Medicine, University Health Network, Toronto, Ontario, Canada

${ }^{8}$ Concussion Care Clinic, University Orthopedics Center, State College, Pennsylvania, USA

${ }^{9}$ Department of Psychology, University of Missouri - Kansas City, Kansas City, Missouri, USA

Acknowledgements The authors wish to acknowledge the critical contributions of the NHL medical personnel (team physicians, athletic therapists/physiotherapists and neuropsychologists) in gathering all data presented herein and their key roles in implementing the NHL/NHLPA Concussion Protocol.

Contributors RJE and JMB assumed full responsibility for all aspects of this study including design, data acquisition, analysis and interpretation of the data, drafting and editing the manuscript, approval of the final version and submission. JT, WM, MGH, JR and PC contributed to the design of the project, data interpretation, manuscript review and editing, and final approval/responsibility of the document.

Funding The authors have not declared a specific grant for this research from any funding agency in the public, commercial or not-for-profit sectors.

Competing interests RJE is a paid consultant for the NHL and co-chair of the NHL/NHLPA Concussion Subcommittee. He is also a paid consultant for Major League Soccer and Princeton University Athletic Medicine. He has a financial interest in EyeGuide and is chair of their Scientific Advisory Board and is currently a co-PI for a grant funded by the NFL (NFL-Long) through Boston Children's Hospital and occasionally provides expert testimony in matters related to MTBI and sports concussion. JT is a part-time employee of the NHL. WM is Medical Director for the NHL and an employee of the NHL. MGH is a member of the NHL/NHLPA Concussion Subcommittee and a consultant to the NHLPA, for which he receives remuneration. JR is a member of the NHL/NHLPA Concussion Subcommittee and a paid medical/ physician consultant to the NHLPA. PC is co-chair of the NHL/NHLPA Concussion Subcommittee and a paid consultant to the NHLPA. JMB is a part-time employee of the NHL. He receives grant funding from Genzyme and is a consultant to Med-IQ and Sporting KC.

Patient and public involvement Patients and/or the public were not involved in the design, or conduct, or reporting, or dissemination plans of this research.

Patient consent for publication Not required.

Ethics approval All data were deidentified and analysed following approval from the University of Missouri-Kansas City Office of Research Compliance (FWA\#00005427).

Provenance and peer review Not commissioned; externally peer reviewed.

Data availability statement All data relevant to the study are included in the article or uploaded as online supplementary information. All aggregate data relevant to the study are included in the article or uploaded as online supplementary information. Individual player raw data are not publicly available.

ORCID iD

Ruben J Echemendia http://orcid.org/0000-0001-6116-8462

\section{REFERENCES}

1 McCrory P, Meeuwisse WH, Echemendia RJ, et al. What is the lowest threshold to make a diagnosis of concussion? Br J Sports Med 2013;47:268-71.

2 Giza CC, Hovda DA. The new neurometabolic cascade of concussion. Neurosurgery 2014;75 Suppl 4:S24-33.
3 Feddermann-Demont N, Echemendia RJ, Schneider KJ, et al. What domains of clinical function should be assessed after sport-related concussion? A systematic review. $\mathrm{Br} J$ Sports Med 2017;51:903-18.

4 McCrory P, Meeuwisse W, Dvorák J, et al. Consensus statement on concussion in sport-the $5^{\text {th }}$ international conference on concussion in sport held in Berlin, October 2016. Br J Sports Med 2017;51:838-47.

5 McCrory P, Feddermann-Demont N, Dvořák J, et al. What is the definition of sportsrelated concussion: a systematic review. Br J Sports Med 2017:51:877-87.

6 Putukian M, Echemendia R, Dettwiler-Danspeckgruber A, et al. Prospective clinical assessment using Sideline concussion assessment Tool-2 testing in the evaluation of sport-related concussion in college athletes. Clin I Sport Med 2015;25:36-42.

$7 \mathrm{McCrory}$ Pet al. Summary and agreement statement of the 2nd International Conference on concussion in sport, Prague 2004. Br J Sports Med 2005:39:i78-86.

8 McCrory P, Meeuwisse W, Johnston K, et al. Consensus statement on concussion in sport: the 3rd International Conference on concussion in sport held in Zurich November 2008. Br J Sports Med 2009;43 Suppl 1:i76-84.

9 Guskiewicz KM, Register-Mihalik J, McCrory P, et al. Evidence-based approach to revising the SCAT2: introducing the SCAT3. Br J Sports Med 2013;47:289-93.

10 Echemendia RJ, Meeuwisse W, McCrory P, et al. The sport concussion assessment tool 5th edition (SCAT5): background and rationale. Br J Sports Med 2017;51:848-50.

11 Echemendia RJ, Broglio SP, Davis GA, et al. What tests and measures should be added to the SCAT3 and related tests to improve their reliability, sensitivity and/ or specificity in sideline concussion diagnosis? A systematic review. Br I Sports Med 2017:51:895-901.

12 Spielberger CD. Assessment of state-trait anxiety: conceptual and methodological issues. The Southern Psychologist 1985:2:6-16.

13 Asken BM, Houck ZM, Bauer RM, et al. SCAT5 vs. SCAT3 symptom reporting differences and convergent validity in collegiate athletes. Arch Clin Neuropsychol 2020;35:291-301.

14 Echemendia RJ, Bruce JM, Meeuwisse W, et al. Can visible signs predict concussion diagnosis in the National hockey League? Br J Sports Med 2018;52:1149-54.

15 Echemendia R, Thelen J, Meeuwisse W, et al. The Use of the Sport Concussion Assessment Tool-5 (SCAT5) in Professional Hockey: Part 1 - Cross-Cultural Normative Data. Manuscript submitted for publication. BJSM.

16 Bruce JM, Echemendia RJ. Concussion history predicts self-reported symptoms before and following a concussive event. Neurology 2004;63:1516-8

17 Ellis M, Krisko C, Selci E, et al. Effect of concussion history on symptom burden and recovery following pediatric sports-related concussion. J Neurosurg Pediatr 2018:21:401-8.

18 Hurtubise JM, Hughes CE, Sergio LE, et al. Comparison of baseline and postconcussion SCAT3 scores and symptoms in varsity athletes: an investigation into differences by sex and history of concussion. BMJ Open Sport Exerc Med 2018:4:e000312.

19 Piland SG, Ferrara MS, Macciocchi SN, et al. Investigation of baseline self-report concussion symptom scores. J Athl Train 2010;45:273-8.

20 Register-Mihalik JK, Mihalik JP, Guskiewicz KM. Association between previous concussion history and symptom endorsement during Preseason baseline testing in high school and collegiate athletes. Sports Health 2009;1:61-5.

21 Downey RI, Hutchison MG, Comper P. Determining sensitivity and specificity of the sport concussion assessment tool 3 (SCAT3) components in University athletes. Brain Inj 2018;32:1345-52.

22 Norheim N, Kissinger-Knox A, Cheatham M, et al. Performance of college athletes on the 10-item word list of SCAT5. BMJ Open Sport Exerc Med 2018:4:e000412.

23 Bailey CM, Echemendia RJ, Arnett PA. The impact of motivation on neuropsychological performance in sports-related mild traumatic brain injury. I Int Neuropsychol Soc 2006;12:475-4.

24 Bailey CM. Effort, invalid performance and the evaluation of sports-related concussion. In: Echemendia RJ, Iverson GL, eds. Oxford Handbook of sports-related concussion. New York, NY: Oxford University Press, 2014.

25 Samadani U, Ritlop R, Reyes M, et al. Eye tracking detects disconjugate eye movements associated with structural traumatic brain injury and concussion. J Neurotrauma 2015;32:548-56.

26 Snegireva N, Derman W, Patricios J, et al. Eye tracking technology in sportsrelated concussion: a systematic review and meta-analysis. Physiol Meas 2018;39:12TR01-28

27 Davis GA, Makdissi M, Bloomfield P, et al. International study of video review of concussion in professional sports. Br J Sports Med 2019;53:1299-304. 